\title{
Neuropathological characteristics of the brain in two patients with SLC19A3 mutations related to the biotin-thiamine-responsive basal ganglia disease
}

\begin{abstract}
Maciej Pronicki ${ }^{1}$, Dorota Piekutowska-Abramczuk², Elżbieta Jurkiewicz ${ }^{3}$, Dariusz Rokicki ${ }^{4}$ Elżbieta Ciara², Joanna Trubicka ${ }^{1}$, Katarzyna Iwanicka-Pronicka ${ }^{5}$, Magdalena Pajdowska ${ }^{6}$, Marek Migdat ${ }^{7}$, Wieslawa A. Grajkowska ${ }^{1,8}$ ${ }^{1}$ Department of Pathology, The Children's Memorial Health Institute, Warsaw, ${ }^{2}$ Department of Medical Genetics, The Children's Memorial Health Institute, Warsaw, ${ }^{3}$ Department of Diagnostic Imaging, The Children's Memorial Health Institute, Warsaw, ${ }^{4}$ Department of Paediatrics, Nutrition and Metabolic Diseases, The Children's Memorial Health Institute, Warsaw, ${ }^{5}$ Department of Audiology and Phoniatrics, The Children's Memorial Health Institute, Warsaw, ${ }^{6}$ Department of Biochemistry, Radioimmunology and Experimental Medicine, The Children's Memorial Health Institute, Warsaw, ${ }^{7}$ Department of Anaesthesiology and Intensive Care, The Children's Memorial Health Institute, Warsaw, ${ }^{8}$ Department of Clinical and Experimental Neuropathology, The Mossakowski Medical Research Centre Polish Academy of Sciences, Warsaw, Poland
\end{abstract}

\begin{abstract}
Biotin-thiamine-responsive basal ganglia disease is a severe form of a rare neurogenetic disorder caused by pathogenic molecular variants in the thiamine transporter gene. Nowadays, a potentially effective treatment is known, therefore the early diagnosis is mandatory. The aim of the paper was to assess the contribution of neuropathological and magnetic resonance imaging (MRI) studies to a proper diagnosis. We present the brain study of two Polish patients with SLC19A3 mutations, including (1) an infant with an intriguing "walnut" appearance of the brain autopsied many years before the discovery of the SLC19A3 defect, and (2) a one-year-old patient with clinical features of Leigh syndrome. In patient 2, biotin/thiamine responsiveness was not tested at the time of diagnosis and causal treatment started with one-year delay.

The central nervous system lesions found in the patients displayed almost clearly a specific pattern for SLC19A3 defect, as previously proposed in diagnostic criteria. Our study presents a detailed description of neuropathological and MRI findings of both patients.

We confirm that the autopsy and/or MRI of the brain is sufficient to qualify a patient with an unknown neuropathological disorder directly for SLC19A3 mutations testing and a prompt trial of specific treatment.
\end{abstract}

Key words: basal ganglia disease, SLC19A3 mutations, MRI, autopsy, neuropathology, thiamine transporter.

\section{Introduction}

A neurogenetic disorder related to homozygous or compound heterozygous mutations in the SLC19A3 gene was firstly described in 2005 [7] and is known by two acronyms: BBGD (biotin-responsive basal ganglia disease) and BTRBGD (biotin-thiamine-respon-

\section{Communicating author}

Prof. Wieslawa A. Grajkowska, Department of Pathology, The Children's Memorial Health Institute, Aleja Dzieci Polskich 20,

04-730 Warsaw, Poland, phone: +48 2281519 60, e-mail: w.grajkowska@ipczd.pl 
sive basal ganglia disease). Since its discovery, almost a hundred patients have been reported, mainly from Saudi Arabia [2], but also from Japan, Lebanon, Mexico, Sweden and other countries. The natural history of the condition is quite well recognized, pointing to a remarkable clinical heterogeneity, even inside the same family and the wide range of severity and prognoses [6]. Recently this has been comprehensively reviewed [1,2].

The distinctive feature of this rare disease is a potential effectiveness of the treatment with biotin and thiamine in high doses $[3,5,18]$. Pathogenic variants in the SLC19A3 [NM_025243.3] coding for thiamine transporter 2 lead to a decrease in the free-thiamine concentration in cells [10]. Brain imaging most commonly shows a basal ganglia involvement of Leigh-like syndrome type $[4,5,15]$ and/or Wernicke encephalopathy features resembling acquired vitamin $B_{1}$ deficiency [7]. Progressive brain atrophy is a relatively common finding [15].

Although in the literature there are numerous magnetic resonance imagining (MRI) series of SLC19A3 defect illustrating both specific changes in detail $[6,17]$ and the response to specific treatment $[1,2]$, only few reports focus on brain pathology in affected patients [15]. Herein, we present our unique autopsy findings ("walnut" appearance of the brain) in a Polish child who died many years ago, and in whom the underlying SLC19A3 homozygous mutation has been recently established by WES (whole-exome sequencing). In the second Polish patient bearing other homozygous SLC19A3 variant we show brain MRI evolution before and after vitamin $B_{1}$ /biotin administration. The treatment which started one year after the disease onset was not sufficiently effective in this child. The aim of the paper was to focus on brain neuropathological findings.

\section{Material and methods}

Neuropathological characteristics of the brain of patients with SLC19A3 defect were analysed in detail to establish usefulness of diagnostic criteria proposed by van den Knaap et al. [6]. Two Polish patients with the SLC19A3 mutations identified out of 113 mitochondrial patients studied in The Children's Memorial Health Institute (CMHI) in the period of 1995-2016 [13] were included.

Routine methods were applied for autopsy study and MRI.
The study was conducted according to the principles of the Helsinki Declaration. The study protocol was approved by the CMHI Bioethics Commission. Informed parental consent was given for the study.

\section{Case reports}

Patient 1 was born spontaneously, from the $3^{\text {rd }}$ uncomplicated pregnancy, with the birth weight of 4050 g; he obtained 8 points in Apgar score. His older sister was healthy, while his brother died at the age of six months due to an acute neurological deterioration. Autopsy was performed but it did not confirm clinically suspected meningitis, however focal cortical dysplasia was described in microscopic evaluation of post-mortem material (specimens not available for verification). The patient displayed global developmental delay, spastic tetraparesis, progressive microcephaly up to much below the $3^{\text {rd }}$ centile (weight 25 c; height 97 c) and seizures since the $3^{\text {rd }}$ month of life.

At the age of 8 months, critical deterioration of the general health condition occurred. General brain atrophy was described in MRI (imaging documentation not available). Blood tests showed metabolic acidosis ( $\mathrm{pH} 7.328$ ) accompanied by normal concentrations of lactate $(1.8 \mathrm{mmol} / \mathrm{l})$ and alanine $(242 \mu \mathrm{mol} / \mathrm{l})$. Cerebrospinal aminoacidogram revealed increased threonine, decreased serine and normal alanine levels. Classic organic acidurias, biotinidase deficiency, aminoacidopathies and lysosomal storage diseases were excluded. The boy died with hypertension, hypothermia, prolonged coma, respiratory and cardiac insufficiency at the age of 8 months, before the SLC19A3 gene discovery. Recently, whole exome sequencing has revealed homozygous SLC19A3 c.74dupT (p.Ser26Leufs*19) mutation. Molecular analysis performed in his family confirmed parental inheritance of the identified variant [13].

Patient 2, a boy was born from the $3^{\text {rd }}$ pregnancy, in term (40 weeks of gestation), with a good clinical status (4350 g; 10 Apgar score). The delivery was complicated by the collarbone fracture and transient hypoxia. The first pregnancy of the mother terminated with intrauterine foetal death. The healthy brother came from the second pregnancy. The patient was under neurological care in infancy due to inappropriate muscle tension, however his motor development was normal. He sat alone between the $7^{\text {th }}-8^{\text {th }}$ month of age, stood and walked with support in the 
$10^{\text {th }}$ month. He started saying his first words on time. At the $12^{\text {th }}$ month of age he stumbled and fell when teething and stopped walking due to hypotonia. The brain MRI revealed basal ganglia changes and diagnosis of Leigh syndrome (LS) was established. Blood and CSF concentrations of lactate were at the upper control range $(2.3$ and $1.7 \mathrm{mmol} / \mathrm{l}$, respectively), biotinidase deficiency was excluded. In the following year, regression in the boy's development was observed, his emotional contact with parents decreased, he stopped standing up and crawled on all fours. Muscle biopsy revealed mitochondrial complex I deficiency (2.8\% of normal value). Further deterioration was observed. This included a complete loss of contact, choking, axial hypotonia with limb hypertonia, strong reflexes, feeding problems requiring a gastric tube and then percutaneous endoscopic gastrostomy (PEG) implantation. Molecular search for the most common LS causative mutations in the SURF1, MTATP6, MTTL1, POLG, SCO2 and MTFMT was negative. At the age of 22 months, a proper diagnosis of BTRBGD associated with SLC19A3 homozygous c.68G $>\mathrm{T}$ (p.Gly23Val) mutation was established by WES [13]. A heterozygous pattern was found in his parents. Vitamin $B_{1}$ and biotin were administered one year after the onset of the disease, in high doses of $200 \mathrm{mg}$ and $5 \mathrm{mg}$ daily (and $300 \mathrm{mg}$ and $10 \mathrm{mg}$ since the age of 4). At the start of treatment the boy demonstrated tetraparesis and severe extrapyrami- dal syndrome with dystonia and sleep-wake phase reversal. Development of brain atrophy was found on second MRI performed two months later. No clear clinical improvement was observed during the vitamin supplementation, but the disease course was without further regression and without exacerbation episodes. Now, at the age of 4, his clinical status is stable, dependent on the type of anticonvulsant treatment with the best response to valproic acid administration (and its good tolerance). Recently, on ketogenic diet, the boy is quieter, has less tensions (spasms) and longer sleep.

\section{Results}

The neuropathological abnormalities found in the patients related to the diagnostic criteria proposed in the literature are shown in Table I. Repeatability of all assessed parameters indicates that the brain neuropathology may present a reliable basis to identify patients with SLC19A3 defect. The detailed findings of autopsy and MRI are described below.

\section{Neuropathological findings on autopsy of patient 1}

Gross examination of formalin fixed brain revealed gyral atrophy of frontal, parietal and occipital lobes, as well as cerebellar hemispheres and vermis (Fig. 1). The degree of gyral atrophy appeared less pronounced in temporal lobes. External examination of the brain

Table I. Topography of neuropathological changes in two patients with SLC19A3 mutations

\begin{tabular}{|c|c|c|}
\hline Topography of changes (according to [3]) & Patient 1 & Patient 2 \\
\hline Method of assessment & Autopsy & MRI \\
\hline Age of assessment & 10 months & 12 months $/ 3$ years \\
\hline Stage of the disease at assessment & End stage & Acute/intermediate \\
\hline Symmetric basal ganglia changes & + & $+1+$ \\
\hline Nucleus caudatus & + & $++/+$ \\
\hline Putamen & + & $++/+$ \\
\hline Globus pallidus & + & $++/+$ \\
\hline Thalamus & + & $++/-$ \\
\hline Cerebral white matter changes & + & $++/-$ \\
\hline Subcortical & + & $+1-$ \\
\hline Central & + & $-1-$ \\
\hline With cortex involvement & + & $+/-$ \\
\hline Cerebellar white matter changes & + & $-1-$ \\
\hline With cortex involvement & + & $-1-$ \\
\hline Abnormalities in the pons & + & $-1-$ \\
\hline In midbrain & + & $+/-$ \\
\hline Atrophy of affected structures on follow-up & Severe & -/Severe \\
\hline
\end{tabular}



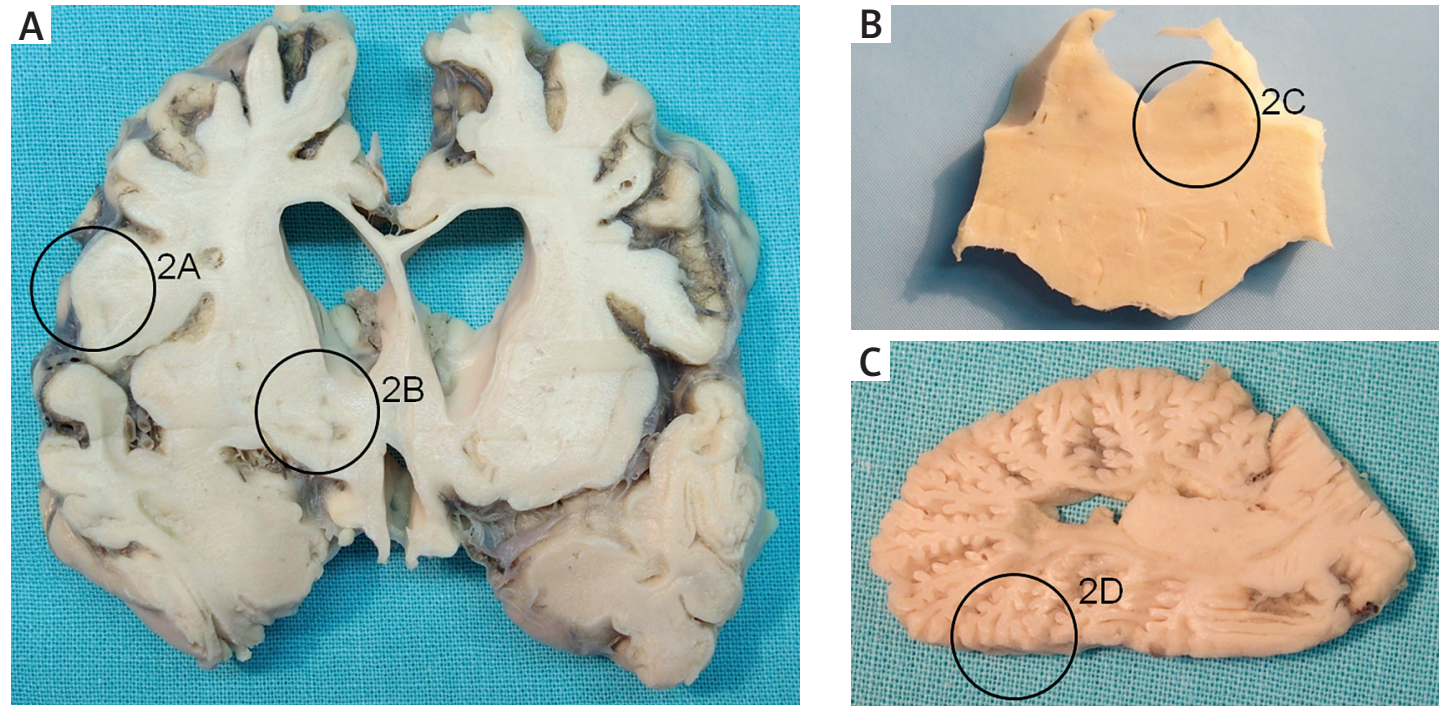

Fig. 1. A) Brain, coronal section showing cortical atrophy, subcortical white matter shrinkage and cavitation, as well as bilateral basal ganglia discoloration and pseudocystic transformation. B) Brain stem, pons. Symmetrical discoloration of subventricular areas. C) Cerebellum with cortical atrophy and subcortical tissue damage. See corresponding histological findings in Figure 2 (areas indicated by circles).

stem structures, including pituitary gland and vasculature, showed no virtual abnormalities. On coronal sections, cerebral and cerebellar cortex appeared to be atrophic in a diffuse manner. Subcortical white matter showed multiple areas of destruction and cavitation. The ventricular system was symmetrically widened. Basal ganglia showed multiple roughly symmetrical areas of pseudocystic destruction. Symmetrical foci of discoloration were found in mesencephalon in the vicinity of cerebral aqueduct, in the floor of the fourth ventricle and dorsal upper medullary region. Discoloration and tissue rarefaction and cavitation encompassed also cerebellar white matter, with accompanying complete blurring of the dentate nuclei.

Microscopic neuropathological examination revealed diffuse bilateral effacement of hemispheric cortical architecture (Fig. 2). Cortex damage included diffuse neuronal loss, pronounced astroglial gemistocytic reaction and focal massive spongiosis with nervous tissue destruction.

Basal ganglia showed necrosis and calcification of multiple neurons with tissue destruction, as well as formation of cavities. Bilateral "Leigh-like" foci of capillary proliferation accompanied by gliosis, spongiosis, oedema and pseudocystic tissue destruction encompassed basal ganglia, periaqueductal mesencephalon, subependymal pontine and medullary areas, as well as cerebellar white matter.
A striking pattern of damage was found in the cerebellar cortex. The most of gyral cerebellar cortical areas showed almost a total loss of neuronal elements. Both external and internal granular layers and Purkinje cells presented multiple areas of wholecell calcifications or complete cell body disappearance. Diffuse areas of replacement glial proliferation occupied most of collapsed, atrophic gyral structures. Only some peripheral "apical” regions of gyri retained some preserved neuronal cortical elements, although also with signs of damage.

Olivary nuclei and spinal cord appeared to be relatively well preserved.

\section{Patient 2 - brain magnetic resonance examination}

Brain MR examination performed at the age of 12 months, during the acute phase, demonstrated swelling of the basal ganglia with cystic degeneration in both lenticular nuclei on T2-weighted images. Bilateral lesions in the crus cerebri, cortex and grey-white matter junction were seen in both cerebral hemispheres. The areas of the cytotoxic oedema were recognized in the cortex and the parts of putamina (Figs. 3A-C). FLAIR image clearly demonstrates cystic degeneration in both lenticular nuclei (Fig. 3D). Diffusion weighted image (Fig. 3E) and ADC 

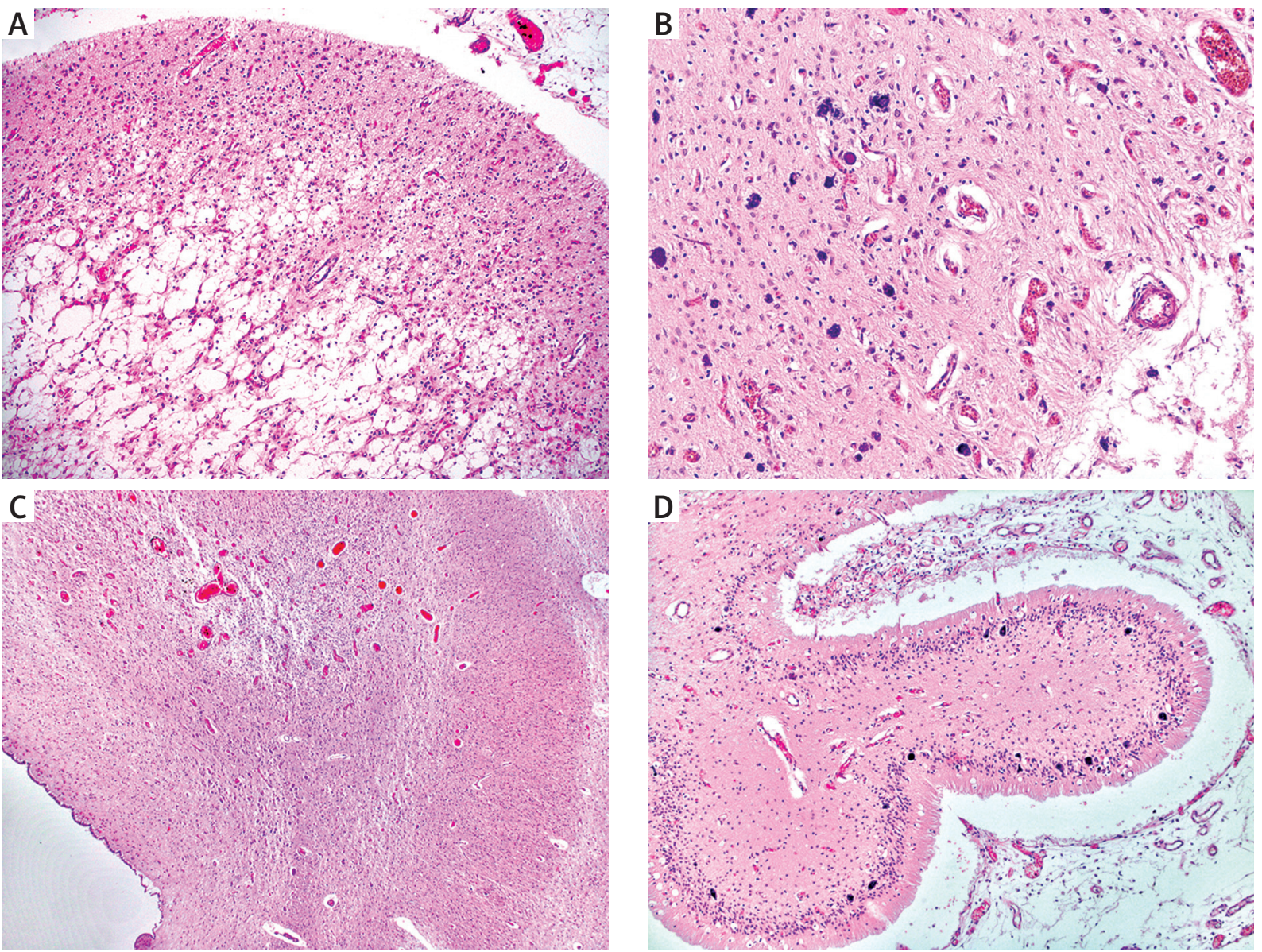

Fig. 2. Central nervous system autopsy material, paraffin sections stained with hematoxylin and eosin.

A) Cerebral cortical and subcortical damage. Original magnification 100x. B) Basal ganglia lesions. Original magnification 200x. C) Brain stem (pons). Leigh-like subependymal focus. Original magnification 40x.

D) Cerebellar cortex atrophy. Original magnification 100x. Detailed description in the text.

map (Fig. 3F) show an increased signal representing vasogenic oedema with multiple areas of restriction diffusion suggesting cytotoxic oedema (Fig. 3F) at both putamen.

Follow-up MR examination at the age of 3 (Fig. 4) revealed generalized infra- and supratentorial cortical and subcortical atrophy (Figs. 4A-D). The signal of both thalami and cerebral cortex normalized. An abnormal signal of the atrophic basal ganglia is decreased and the size of cystic lesions are diminished (Figs. 4A-C). Interestingly, in contrast to patient 1, the cerebellar cortex showed no detectable atrophic changes.

\section{Discussion}

In both studied cases, the diagnosis of SLC19A3 defect was established too late to reliably check the effectiveness of biotin/thiamine administration. Intriguing specificity of brain morphology of "walnut pattern" [8] found at autopsy in patient 1 was the cause of the return to investigation after years, with the advent of the whole exome sequencing, which finally unravelled the disease cause. In patient 2, Leigh syndrome was diagnosed by MRI at onset (in 2013) but SLC19A3 defect was not considered and therefore biotin/thiamine administration was not tested then. After establishing molecular diagnosis of SLC19A3 defect, the first MRI was re-assessed and found typical of BTRBGD.

In 2013, the research team of van den Knaap identified a distinct MRI pattern [6] in seven patients with lethal leukoencephalopathy (out of more than 3000 MRIs). Using WES technology they linked this condition to SLC19A3 mutations. The pattern includ- 


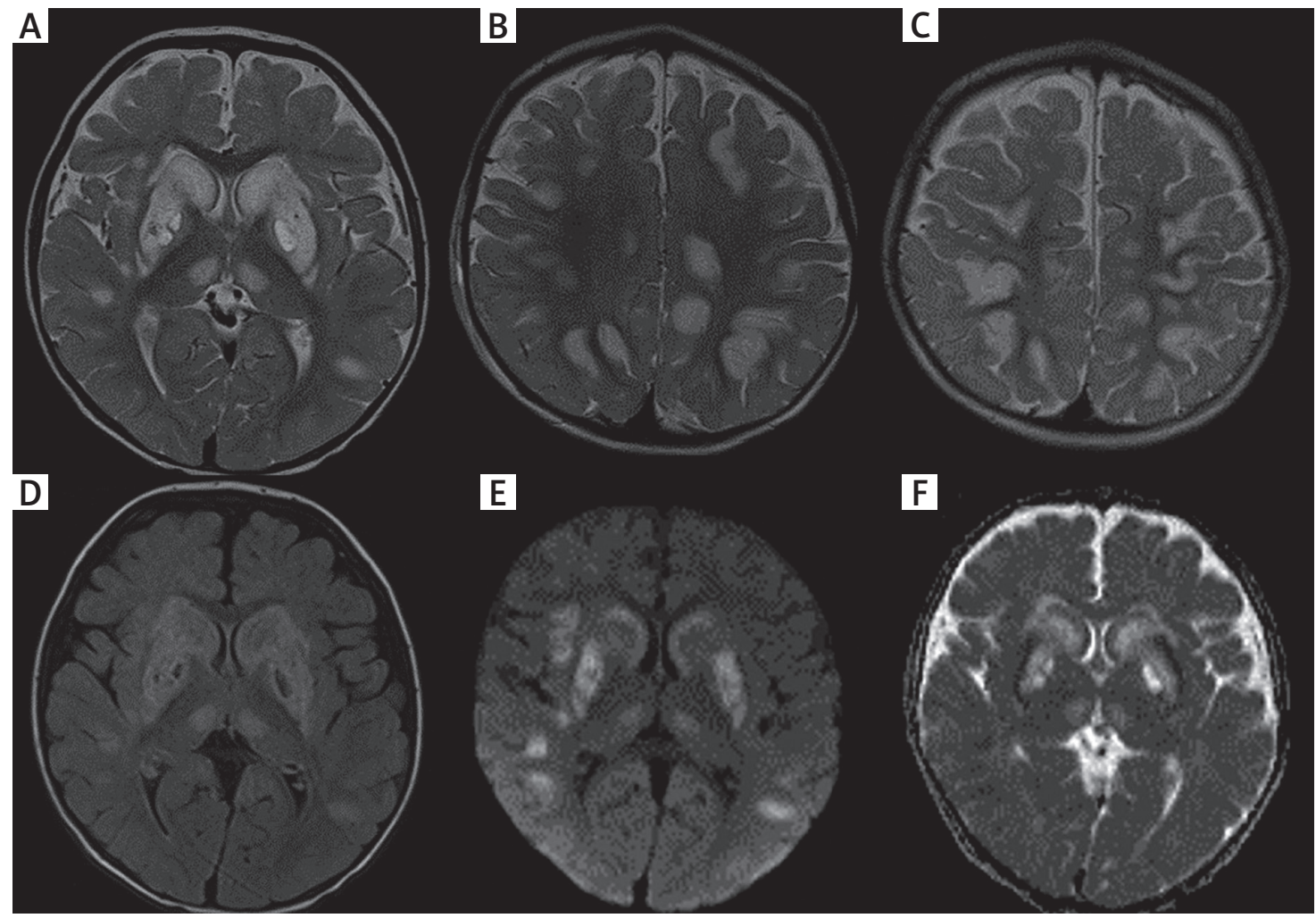

Fig. 3. Brain magnetic resonance imaging at the age of 12 months, the acute phase. Axial T2-weighted images demonstrate a bilateral and symmetric increased signal of the head of the caudate nuclei, putamina, globus pallidi and medial parts of both thalami (A and D). Abnormal T2-hyperintensities of the cerebral cortex and grey-white matter junction in both cerebral hemispheres (B and C). Axial FLAIR image (D) clearly demonstrates cystic degeneration of both lenticular nuclei. Diffusion weighted image (E) and ADC map (F) show an increased signal and represents vasogenic oedema with areas of restriction diffusion (F) suggesting cytotoxic oedema in parts of both putamina.
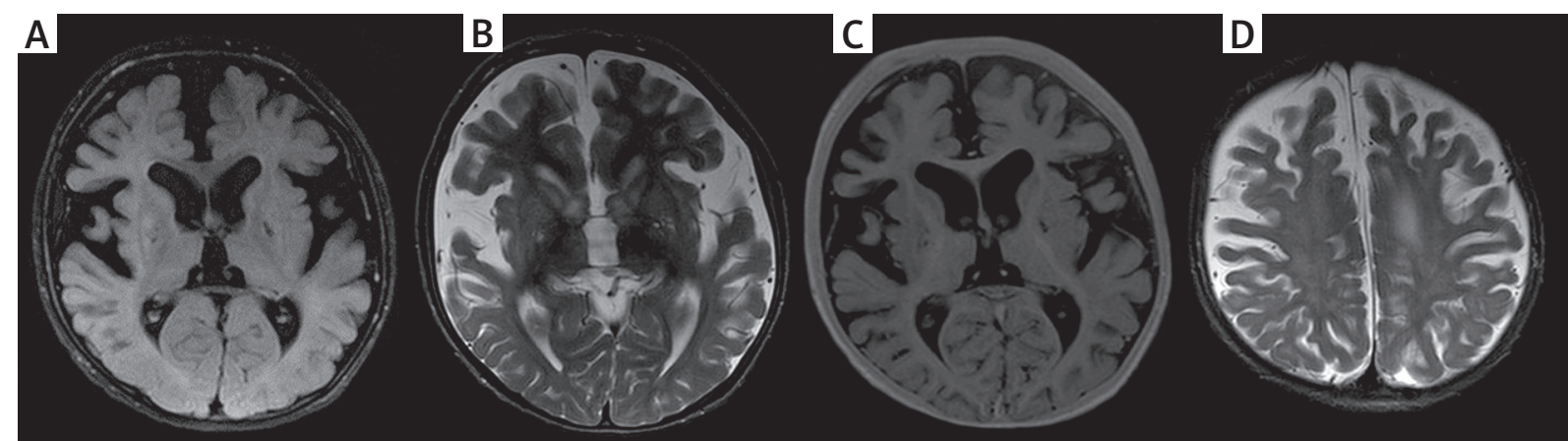

Fig. 4. Follow-up brain magnetic resonance at the age of 3 years. Generalized cortical and subcortical brain atrophy is seen (A-D). The signal of both thalami and cerebral cortex is normalized. Abnormal signal of the atrophic basal ganglia is decreased and the size of cystic lesions is diminished (A-C).

ed: "(i) bilateral signal abnormalities of the nucleus caudatus, putamen, globus pallidus and thalamus; (ii) extensive signal abnormalities of the subcortical and central cerebral white matter and the cerebral cortex; (iii) diffuse signal abnormalities of the cerebellar white matter with or without involvement of the cortex; (iv) extensive signal abnormalities in the pons and midbrain; and (v) in the case of follow-up 
MRIs, atrophy of affected structures" [6]. Acute, postacute, intermediate and end stage of the disease were specified in that study [6]. Up to now more than 75 patients were reported and natural history of the disease was presented in detail [10].

The clinical course of both presented patients demonstrated the most severe type of the SLC19A3 defect. Neuropathological findings were in full accordance with the diagnostic criteria (Table I) for the disease [6]. We have to emphasize that in both patients a mitochondrial disease in the form of Leigh disease was also expressed, with typical topography and typical histopathological features of capillary proliferation, gliosis, spongiosis, oedema and pseudocystic formation in the affected brain regions, as described first by David Leigh in 1954 [9].

Our detailed description of human brain histology of SLC19A3 defect is the second report, after the first one [6], which showed almost the same neuropathological damage pattern. Interestingly, there is also an animal model of the disease, studied in detail [16]. The dogs, Alaskan Huskies, demonstrated similar histological features to the affected humans. It included bilateral symmetrical, cavitary, malacic lesions in the thalamus, putamen, claustrum, junctional grey and white matter in the cerebral cortex, brainstem and midline cerebellar vermis [9]. Importantly, an occurrence of some interspecies differences was noticed by the authors [16].

About twenty five mutations including these identified in our study have been reported until today in the SLC19A3 gene encoding a ubiquitously expressed human thiamine transporter 2 (hTHTR2). Most of them are missense/nonsense changes [HGMD Professional 2016.4].

The c.74dupT variant found in patient 1 is predicted to result in a frameshift starting at codon Ser26 and premature termination of protein translation (Ser26Leufs ${ }^{\star 19}$ ). This mutation was reported twice $[3,14]$ in the compound heterozygous state.

The c.68G $>$ T variant identified in patient 2 in the homozygous state resulted in substitution of highly evolutionary conserved Gly23 into Val. It is a very rare variant as its minor allele frequency (MAF) reported in the Exome Aggregation Consortium (ExAC) database is 0.000008 . It was found up to now in three other families $[6,10,12,19]$ including two with the same c.68G >T/c.68G>T genotype [19] as patient 2 . One of them, Yemeni originated, presented first symptoms at the age of 12 months, just like our patient 2. The biotin supplementation was started immediately (in 4 days) and its effectiveness was very good $[11,19]$. The second reported patient with a homozygous c.68G $>T$ variant, of Spanish origin, presented with acute encephalopathy and lactic acidosis during early infancy and also responded quickly to the biotin/thiamine treatment [12], however, on the follow-up observation, brain necrotic changes and cerebral atrophy developed in this patient.

In summary, our observation confirmed that morphological characteristics of the brain (autopsy or MRI) are quite specific to suspect BTRBGD and to start a cascade family study and/or test of biotin/thiamine administration immediately, before the final molecular identification of SLC19A3 defect.

\section{Acknowledgements}

Funding: S136/13, S238/16, 2012/05/B/NZ2/ 01627, N407 075137.

\section{Disclosure}

Authors report no conflict of interest.

\section{References}

1. Alfadhel M, Almuntashri M, Jadah RH, Bashiri FA, Al Rifai MT, Al Shalaan H, Al Balwi M, Al Rumayan A, Eyaid W, Al-Twaijri W. Biotin-responsive basal ganglia disease should be renamed biotin-thiamine-responsive basal ganglia disease: a retrospective review of the clinical, radiological and molecular findings of 18 new cases. Orphanet J Rare Dis 2013; 8: 83.

2. Algahtani H, Ghamdi S, Shirah B, Alharbi B, Algahtani R, Bazaid A. Biotin-thiamine-responsive basal ganglia disease: catastrophic consequences of delay in diagnosis and treatment. Neurol Res 2017; 39: 117-125.

3. Debs R, Depienne C, Rastetter A, Bellanger A, Degos B, Galanaud D, Keren B, Lyon-Caen O, Brice A, Sedel F. Biotin-responsive basal ganglia disease in ethnic Europeans with novel SLC19A3 mutations. Arch Neurol 2010; 67: 126-130.

4. Fassone E, Wedatilake Y, DeVile CJ, Chong WK, Carr LJ, Rahman S. Treatable Leigh-like encephalopathy presenting in adolescence. BMJ Case Rep 2013; 2013: 200838.

5. Haack TB, Klee D, Strom TM, Mayatepek E, Meitinger T, Prokisch H, Distelmaier F. Infantile Leigh-like syndrome caused by SLC19A3 mutations is a treatable disease. Brain 2014; 137 : e295.

6. Kevelam SH, Bugiani M, Salomons GS, Feigenbaum A, Blaser S, Prasad C, Häberle J, Baric I, Bakker IM, Postma NL, Kanhai WA, Wolf NI, Abbink TE, Waisfisz Q, Heutink P, van der Knaap MS. Exome sequencing reveals mutated SLC19A3 in patients with an early-infantile, lethal encephalopathy. Brain 2013; 136: 15341543. 
7. Kono S, Miyajima H, Yoshida K, Togawa A, Shirakawa K, Suzuki H. Mutations in a thiamine-transporter gene and Wernicke's-like encephalopathy. N Engl J Med 2009; 360: 1792-1794.

8. Laurence KM, Cavanagh JB. Progressive degeneration of the cerebral cortex in infancy. Brain 1968; 91: 261-280.

9. Leigh D. Subacute necrotizing encephalomyelopathy in an infant. J Neurol Neurosurg Psychiatry 1951; 14: 216-221.

10. Ortigoza-Escobar JD, Molero-Luis M, Arias A, Oyarzabal A, Darín N, Serrano M, Garcia-Cazorla A, Tondo M, Hernández M, GarciaVilloria J, Casado M, Gort L, Mayr JA, Rodríguez-Pombo P, Ribes A, Artuch R, Pérez-Dueñas B. Free-thiamine is a potential biomarker of thiamine transporter-2 deficiency: a treatable cause of Leigh syndrome. Brain 2016; 139: 31-38.

11. Ozand PT, Gascon GG, Al Essa M, Joshi S, Al Jishi E, Bakheet S, Al Watban J, Al-Kawi MZ, Dabbagh O. Biotin responsive basal ganglia disease: a novel entity. Brain 1998; 121: 1267-1279.

12. Pérez-Dueñas B, Serrano M, Rebollo M, Muchart J, Gargallo E, Dupuits C, Artuch R. Reversible lactic acidosis in a newborn with thiamine transporter-2 deficiency. Pediatrics 2013; 131: e1670-5.

13. Pronicka E, Piekutowska-Abramczuk D, Ciara E, Trubicka J, Rokicki D, Karkucińska-Więckowska A, Pajdowska M, Jurkiewicz E, Halat P, Kosińska J, Pollak A, Rydzanicz M, Stawinski P, Pronicki M, Krajewska-Walasek M, Płoski R. New perspective in diagnostics of mitochondrial disorders: two years' experience with whole-exome sequencing at a national paediatric centre. J Transl Med 2016; 14: 174.

14. Sremba LJ, Chang RC, Elbalalesy NM, Cambray-Forker EJ, Abdenur JE. Whole exome sequencing reveals compound heterozygous mutations in SLC19A3 causing biotin-thiamine responsive basal ganglia disease. Mol Genet Metab Rep 2014; 1: 368-372.

15. van der Knaap MS, Kevelam SH. Reply: Infantile Leigh-like syndrome caused by SLC19A3 mutations is a treatable disease. Brain 2014; 137: e297.

16. Vernau KM, Runstadler JA, Brown EA, Cameron JM, Huson HJ, Higgins RJ, Ackerley C, Sturges BK, Dickinson PJ, Puschner B, Giulivi C, Shelton GD, Robinson BH, DiMauro S, Bollen AW, Bannasch DL. Genome-wide association analysis identifies a mutation in the thiamine transporter 2 (SLC19A3) gene associated with Alaskan Husky encephalopathy. PLoS One 2013; 8: e57195.

17. Yamada K, Miura K, Hara K, Suzuki M, Nakanishi K, Kumagai T, Ishihara N, Yamada Y, Kuwano R, Tsuji S, Wakamatsu N. A wide spectrum of clinical and brain MRI findings in patients with SLC19A3 mutations. BMC Med Genet 2010; 11: 171.

18. Ygberg S, Naess K, Eriksson M, Stranneheim H, Lesko N, Barbaro M, Wibom R, Wang C, Wedell A, Wickström R. Biotin and thiamine responsive basal ganglia disease - a vital differential diagnosis in infants with severe encephalopathy. Eur J Paediatr Neurol 2016; 20: 457-461.

19. Zeng WQ, Al-Yamani E, Acierno JS Jr, Slaugenhaupt S, Gillis T, MacDonald ME, Ozand PT, Gusella JF. Biotin-responsive basal ganglia disease maps to 2 q36.3 and is due to mutations in SLC19A3. Am J Hum Genet 2005; 77: 16-26. 